\title{
Low-temperature technique of thin silicon ion implanted epitaxial detectors
}

\author{
A.J. Kordyasz ${ }^{1, a}$, N. Le Neindre ${ }^{2}$, M. Parlog ${ }^{2,3}$, G. Casini ${ }^{4}$, R. Bougault ${ }^{2}$, G. Poggi ${ }^{4}$, A. Bednarek $^{1}$, M. Kowalczyk $^{1,5}$, \\ O. Lopez $^{2}$, Y. Merrer ${ }^{2}$, E. Vient ${ }^{2}$, J.D. Frankland ${ }^{6}$, E. Bonnet ${ }^{6}$, A. Chbihi ${ }^{6}$, D. Gruyer 6 , B. Borderie ${ }^{7}$, G. Ademard ${ }^{7}$, \\ P. Edelbruck ${ }^{7}$, M.F. Rivet ${ }^{7}$, F. Salomon ${ }^{7}$, M. Bini ${ }^{4}$, S. Valdré ${ }^{4}$, E. Scarlini ${ }^{4}$, G. Pasquali ${ }^{4}$, G. Pastore ${ }^{4}$, S. Piantelli $^{4}$, \\ A. Stefanini ${ }^{4}$, A. Olmi ${ }^{4}$, S. Barlini ${ }^{4}$, A. Boiano ${ }^{8}$, E. Rosato ${ }^{8}$, A. Meoli ${ }^{8}$, A. Ordine ${ }^{8}$, G. Spadaccini ${ }^{8}$, G. Tortone $^{8}$, \\ M. Vigilante ${ }^{8}$, E. Vanzanella ${ }^{8}$, M. Bruno ${ }^{9}$, S. Serra ${ }^{9}$, L. Morelli ${ }^{9}$, M. Guerzoni ${ }^{9}$, R. Alba ${ }^{10}$, D. Santonocito $^{10}$, \\ C. Maiolino ${ }^{10}$, M. Cinausero ${ }^{11}$, F. Gramegna ${ }^{11}$, T. Marchi ${ }^{11}$, T. Kozik ${ }^{12}$, P. Kulig ${ }^{12}$, T. Twaróg ${ }^{12}$, Z. Sosin ${ }^{12}$, \\ K. Gạsior ${ }^{13}$, A. Grzeszczuk ${ }^{13}$, W. Zipper ${ }^{13}$, J. Sarnecki ${ }^{14}$, D. Lipiński ${ }^{14}$, H. Wodzińska ${ }^{14}$, A. Brzozowski ${ }^{14}$,

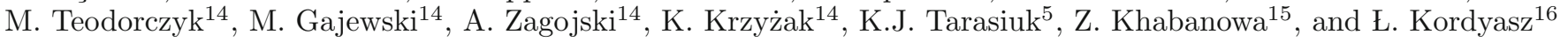 \\ 1 Heavy Ion Laboratory, Warsaw University, Warsaw, Poland \\ 2 LPC, IN2P3-CNRS, ENSICAEN and Université de Caen, F-14050 Caen-Cedex, France \\ 3 "Horia Hulubei" National Institute of Physics and Nuclear Engineering (IFIN-HH), RO-077125 Bucharest Magurele, Romania \\ 4 INFN and Università di Firenze, via G. Sansone 1, 50019 Sesto Fiorentino (Firenze), Italy \\ 5 Institute of Experimental Physics, University of Warsaw, Warsaw, Poland \\ ${ }^{6}$ GANIL, CEA and IN2P3-CNRS, B.P. 5027, 14076 Caen-Cedex 05, France \\ 7 Institut de Physique Nucléaire, IN2P3-CNRS, 91406 Orsay-Cedex, France \\ 8 INFN and Dipartimento di Scienze Fisiche, Università di Napoli "Federico II", Napoli, Italy \\ 9 INFN and Università di Bologna, 40126 Bologna, Italy \\ 10 LNS, INFN and Università di Catania, 95129 Catania, Italy \\ 11 INFN LNL Legnaro, viale dell'Università 2, 35020 Legnaro (Padova), Italy \\ 12 Jagiellonian University, Cracow, Poland \\ 13 Silesian University, University of Silesia, Katowice, Poland \\ 14 Institute of Electronic Materials Technology, Warsaw, Poland \\ 15 Warsaw University of Technology, Faculty of Physics, Warsaw, Poland \\ 16 Warsaw University of Technology, Faculty of Mechatronics, Institute of Mikromechanics and Photonics, Department of Design \\ of Precision Devices, Warsaw, Poland
}

Received: 30 October 2014 / Revised: 15 January 2015

Published online: 9 February 2015

(C) The Author(s) 2015. This article is published with open access at Springerlink.com

Communicated by H. Miyatake

\begin{abstract}
A new technique of large-area thin ion implanted silicon detectors has been developed within the R\&D performed by the FAZIA Collaboration. The essence of the technique is the application of a lowtemperature baking process instead of high-temperature annealing. This thermal treatment is performed after $\mathrm{B}^{+}$ion implantation and $\mathrm{Al}$ evaporation of detector contacts, made by using a single adjusted Al mask. Extremely thin silicon pads can be therefore obtained. The thickness distribution along the $X$ and $Y$ directions was measured for a prototype chip by the energy loss of $\alpha$-particles from ${ }^{241} \mathrm{Am}$ $\left(\left\langle E_{\alpha}\right\rangle=5.5 \mathrm{MeV}\right)$. Preliminary tests on the first thin detector (area $\approx 20 \times 20 \mathrm{~mm}^{2}$ ) were performed at the INFN-LNS cyclotron in Catania (Italy) using products emitted in the heavy-ion reaction ${ }^{84} \operatorname{Kr}(E=$ $35 \mathrm{AMeV})+{ }^{112} \mathrm{Sn}$. The $\Delta E-E$ ion identification plot was obtained using a telescope consisting of our thin $\Delta E$ detector $(21 \mu \mathrm{m}$ thick) followed by a typical FAZIA $510 \mu \mathrm{m}$ E detector of the same active area. The charge distribution of measured ions is presented together with a quantitative evaluation of the quality of the $Z$ resolution. The threshold is lower than $2 \mathrm{AMeV}$ depending on the ion charge.
\end{abstract}

\footnotetext{
${ }^{a}$ e-mail: kord@slcj.uw.edu.pl
} 


\section{Introduction}

The FAZIA Collaboration performed important activity for testing and improving the performance of silicon detectors to be used for heavy-ion physics [1]. In many modern experiments the identification of the charge and possibly the mass of each detected fragment is crucial. Indeed, the full reconstruction of the events, often associated with a large variety of products with different energies, is necessary to try an accurate description of the reaction mechanisms and in particular of the nucleon exchange during the interaction. For this purpose, the FAZIA Collaboration have performed an intense research and development study, optimizing both the detector construction and the associated electronics. Specifically, efforts have been done to study the Pulse Shape Analysis (PSA) in silicons for identification of stopped heavy ions, to investigate the influence of channeling effects [2], to test the worsening due to radiation damage [3] and to implement non-destructive methods for mapping the resistivity on silicon pads [4]. Moreover, we developed new electronics, both the analogue stages based on high-quality charge and currentsensitive preamplifiers [5] and the subsequent processing circuits capable of fast real-time analysis and transfer of the sampled data streams [6-8]. It has been shown that particle identification thresholds are significantly reduced using the PSA with respect to standard $\Delta E-E$ technique for $300 \mu \mathrm{m}$ front Si detectors, provided that highquality detectors (in reverse mounting configuration) and electronics are employed [9-11]. Expressed as a function of the range in silicon, particle (charge) identification thresholds resulted to be around $30 \mu \mathrm{m}$ for $Z=5$, growing up to $150 \mu \mathrm{m}$ for $Z=40$ [9-11].

The motivation of this work is an attempt to decrease particle identification thresholds by strongly reducing the thickness of the silicon front detector (down to about $20 \mu \mathrm{m}$ ) but keeping relatively large active areas, thus recovering the $\Delta E-E$ technique of the telescope configuration.

The technology of ion implanted silicon detectors has been known for a long time [12]. Application of microelectronics achievements as silicon wafer polishing, thermal oxidation — needed to reduce the leakage currentand photolithography was introduced by Kemmer [13] to the detector technology. As a result, low-noise passivated silicon detectors were successfully produced by using this progress in silicon technology. However, the application of the Kemmer procedure to thin silicon films - obtained by anodic dissolution of the thick substrate by means of a $5 \%$ aqueous HF solution jet [14] — is very difficult, since the attempt of a subsequent thermal oxidation (at $1030^{\circ} \mathrm{C}$ in ref. [13]) of the thin silicon membrane, destroys it due to thermal stresses. An alternative method to make low-noise thin silicon passivated detectors featured a two-step process $[15,16]$. The first step is an application of planar process as developed by Kemmer [13] to the epitaxial side of the $n^{+}-n$ structure; this step includes the doping with $\mathrm{B}^{+}$ions for the $\mathrm{p}^{+}$layer creation. The second step is an electro-chemical thinning by anodic dissolution of the detector substrate followed by $\mathrm{Al}$ deposition of the back detector contact $[15,16]$. The essential problem is the protection of the detector oxide layers against the very aggressive $\mathrm{HF}$ vapour accompanying the dissolution process which can destroy the $\mathrm{SiO}_{2}$ layer. This problem has been overcome by using a hermetic detector housing attached to the silicon detector by epoxy glue. The top cover of the detector housing is sealed with a rubber o-ring against $\mathrm{HF}$ vapor and it is removable for $\Delta E-E$ experiments. This solution indeed permitted to reach very good identification for the measured heavy ions $[15,16]$. However, the largest surface obtained in this way was of $50 \mathrm{~mm}^{2}$. This solution is not suitable for the FAZIA standard square detector frames which accept $21.6 \times 21.6 \mathrm{~mm}^{2}$ silicon plates with active area of $20 \times 20 \mathrm{~mm}^{2}$. The production of thin silicon detectors having the FAZIA size with the above-described technology $[15,16]$ is extremely difficult from the technological point of view, since the dead detector border is less than $1 \mathrm{~mm}$. In addition, the sequence of the operations in this method $[15,16]$ does not allow manufacturing strip detectors since the speed of the anodic dissolution process is faster for the strip regions with $\mathrm{B}^{+}$ion implantation than for the interstrip regions where no doping implantation exists at all. As a result of the anodic dissolution, a non-uniform thickness of strip detector is obtained [14]. The next solution for producing passivated thin silicon detectors is the planar process partially performed on the thin silicon membrane (PPPP process) [17]. In this case the initial thermal oxidation and opening of windows by photolithography are performed on the $n^{+}-n$ thick wafer by standard Kemmer technology [13] while only the processes like $\mathrm{B}^{+}$ion implantation and photolithography with Al layer are performed on the thin silicon membrane after the anodic dissolution of $\mathrm{n}^{+}$substrate. Again, however, these last technological steps of the PPPP process are very difficult to be performed on the delicate thin silicon membrane.

The aim of the present work is the implementation of a feasible thin silicon detector technique avoiding photolithography which is the risky operation to be performed on the fragile, thin silicon membrane.

\section{Low-temperature technique of thin silicon ion implanted epitaxial detectors}

The new technique to obtain thin silicon epitaxial ion implanted detectors is illustrated in fig. 1. We started from a silicon epitaxial structure $\mathrm{n}^{+}-\mathrm{n}$, with a resistivity of about $900 \Omega \mathrm{cm}$ and thickness $21 \mu \mathrm{m}$, epitaxially grown on a thick $(400 \mu \mathrm{m}),\langle 111\rangle$-oriented, low-resistivity $(0.01 \Omega \mathrm{cm}) \mathrm{n}^{+}$substrate (upper part of the figure). The sample has been produced at the Institute of Electronic Materials Technology, Warsaw, Poland. Then the substrate of the structure $\mathrm{n}^{+}-\mathrm{n}$ was removed by anodic dissolution using a HF jet [14] (middle part of the figure). The external part of the substrate close to the wafer edges is not removed in the process and acts as a mechanical support of the thin silicon epitaxial membrane. The top of 
High resistivity, thin, $\mathrm{n}$-type $\mathrm{Si}$ epitaxial layer

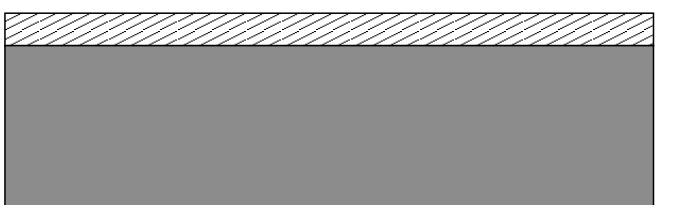

21 micron

400

microns

Low resistivity, $\mathrm{n}^{+}-$type thick $\mathrm{Si}$ substrate



$50 \mathrm{keV}$ Boron ions implantation followed by $\mathrm{Al}$ evaporation

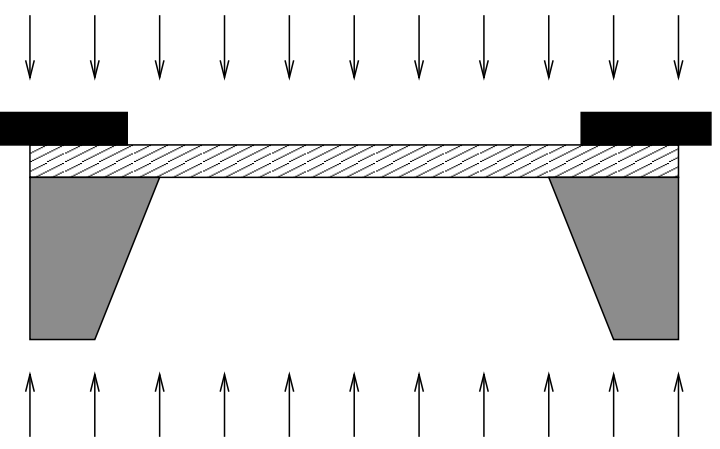

Al evaporation followed by long time baking of the Si detector

Fig. 1. Technology of thin silicon detectors. The starting $n^{+}-n$ silicon structure is shown in the upper part of the figure. The low-resistivity, thick $\mathrm{n}^{+}$-type substrate is removed by the anodic dissolution (central part of the figure), then $50 \mathrm{keV} \mathrm{B}{ }^{+}$ ion implantation is performed followed by Al metallization on both sides of the n-type epitaxial thin silicon membrane.

the etched silicon epitaxial structure $n^{+}-n$ has been further collimated by means of an $\mathrm{Al}$ mask (see the bottom part of the figure) in order to select the region of the epitaxial layer for the further $50 \mathrm{keV} \mathrm{B}{ }^{+}$ion implantation with a fluence of $5 \times 10^{14}$ ions $/ \mathrm{cm}^{2}$. After boron implantation, final Al layers are deposited on both silicon faces, also on the front one where the Al-mask is already there. The $\mathrm{B}^{+}$ion implantation within the silicon epitaxial layer produces a $\mathrm{p}^{+}-\mathrm{n}$ junction while the evaporated $\mathrm{Al}$ film creates the electric contact both on the $\mathrm{p}^{+}$implanted side of the detector and on the rear wafer side, (fig. 1, lower panel). In order to activate the $\mathrm{p}^{+}-\mathrm{n}$ junction, a final baking step was applied in air in an oven at $160^{\circ} \mathrm{C}$ for three days. This long-time, lowtemperature, baking process is applied instead of the standard short time (about one hour), high-temperature (600-950) ${ }^{\circ} \mathrm{C}$ post-implantation annealing in inert atmosphere.
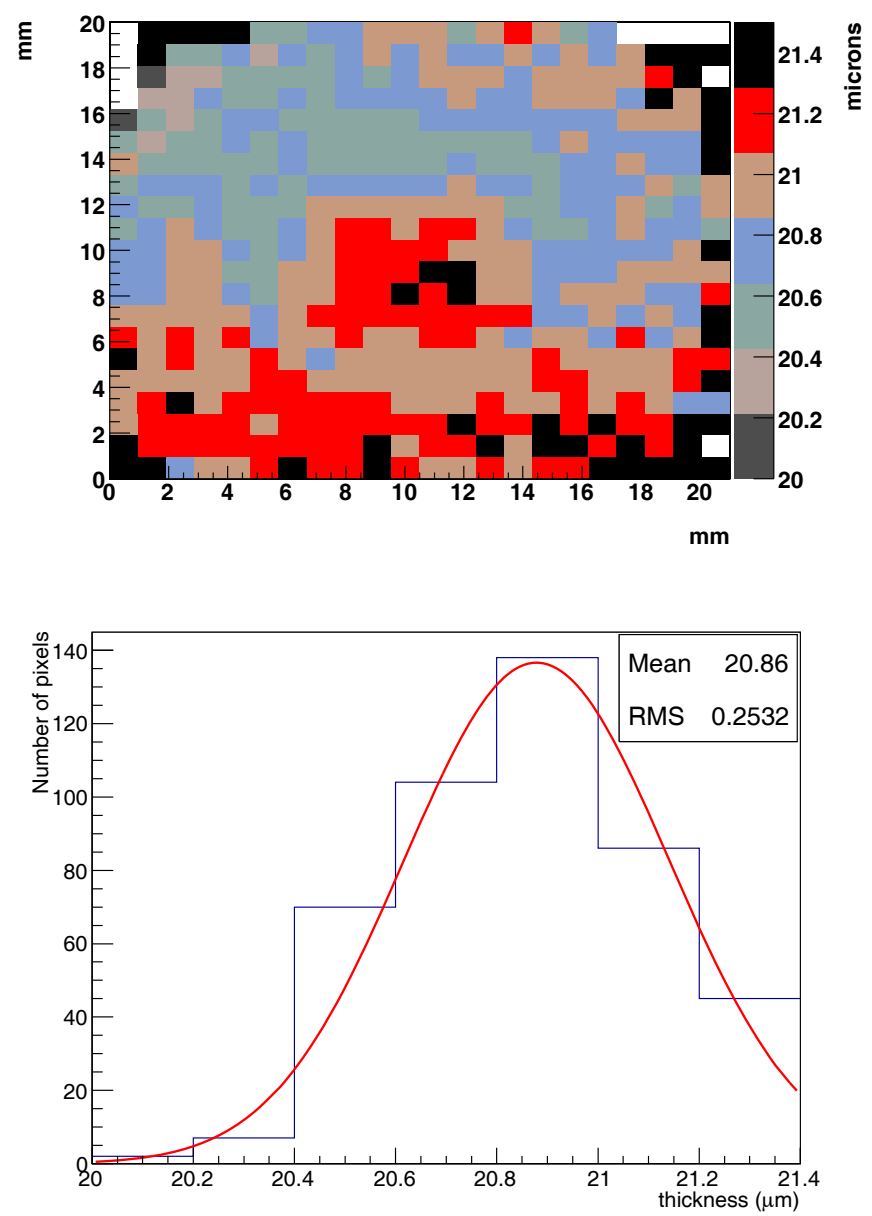

Fig. 2. (Color online) Thickness distribution of the epitaxial thin silicon detector (upper panel) and the corresponding histogram (lower panel). Measurements have been performed using an $\alpha$-particle ${ }^{241} \mathrm{Am}$ source and an average thickness of $21 \mu \mathrm{m}$ has been found.

The thin detector thickness pattern (with one mm steps in the $X$ and $Y$ directions) was measured by transmission of $\alpha$-particles from a ${ }^{241} \mathrm{Am}\left(\left\langle E_{\alpha}\right\rangle=5.5 \mathrm{MeV}\right)$ source using a PIN diode as a stop detector and the result is shown in fig. 2. The thickness distribution was calculated using range-energy tables of $\alpha$-particles in silicon. The average thickness resulting from the map of fig. 2 is $\approx 21 \mu \mathrm{m}$ and the non-uniformity is of about $1 \mu \mathrm{m}$. The thickness map has approximately a central symmetry (with an island in the center). It is related to the method of the anodic dissolution process [14], probably due to a too high anodic dissolution electric current at the end of the process. According to the opinion of Lipiński, the manufacturer of silicon epitaxial $n^{+}-n$ structures, the thickness non-uniformity is usually well below $1 \mu \mathrm{m}$ over dimensions of around $20 \mathrm{~mm}$. For this reason we hope that, by improving our method of dissolution process, we can obtain more uniform silicon epitaxial membranes. 


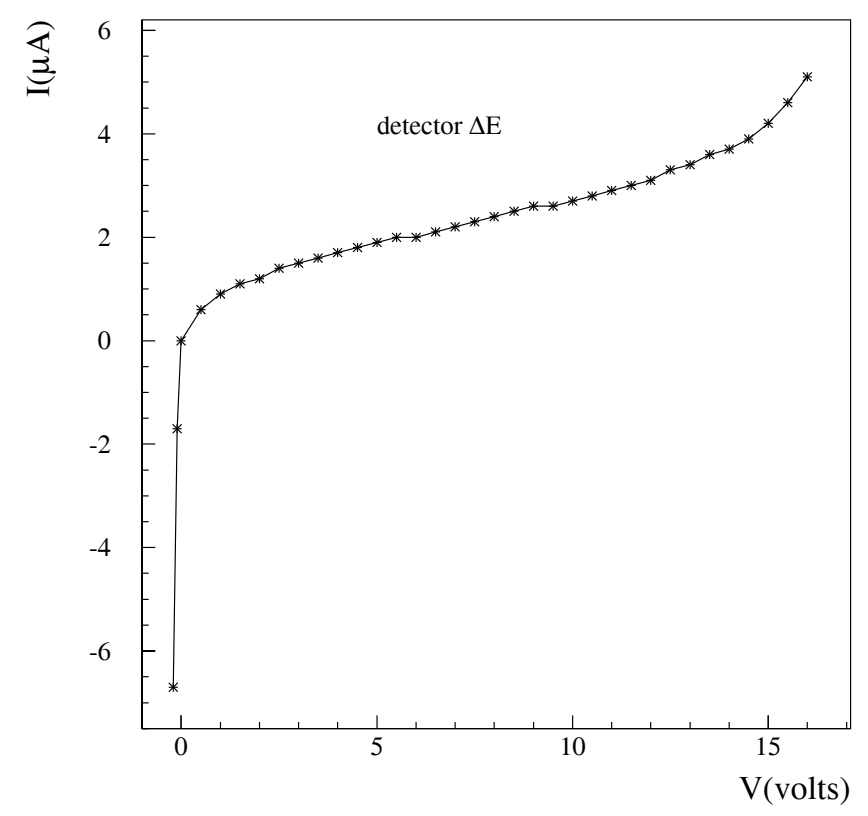

Fig. 3. Voltage-current characteristic of the epitaxial $21 \mu \mathrm{m}$ silicon detector. The two leftmost points describe forward current with low direct bias voltage.

\section{Under beam test}

The thin $\approx 20 \times 20 \mathrm{~mm}^{2}$ detector (Si1) built as described above and with voltage-current characteristics presented in fig. 3 has been mounted in a $\Delta E(\mathrm{Si} 1)-E(\mathrm{Si} 2)$ telescope configuration as the first $\Delta E(\mathrm{Si1})$ stage, followed by a $510 \mu \mathrm{m} E(\mathrm{Si} 2)$ thick silicon detector. The $E(\mathrm{Si} 2)$ is a typical silicon pad of the FAZIA Collaboration [10]. A CsI(Tl) scintillator detector read by a photodiode was mounted as a third layer in the telescope and it has been used in the present analysis as a veto to remove the particles punching through the two silicon stages. The geometrical sketch of the three stage telescope: $\mathrm{Si} 1, \mathrm{Si} 2$ and $\mathrm{CsI}(\mathrm{Tl})$ is illustrated in fig. 4. Each detector was coupled to a low-noise PACI preamplifier [5]; the charge and current signals from the PACI, were sent to digital fast sampling stages designed and used by the FAZIA group for the test experiments. The details of the electronics are described elsewhere [6$8]$. We mention here that the charge signals were sampled via a fast $\mathrm{ADC}$ and then shaped by means of a software trapezoidal filter with $2 \mu$ s rise time and $1 \mu$ s flat top for both $\Delta E(\mathrm{Si} 1)$ and $E(\mathrm{Si} 2)$ detectors. For the $\mathrm{CsI}(\mathrm{Tl}), 2 \mu \mathrm{s}$ rise time and $16 \mu \mathrm{s}$ flat top were used. Preliminary tests of this telescope were conducted at CS cyclotron of the INFN-LNS in Catania (Italy) using fragments produced in the heavy-ion reaction ${ }^{84} \mathrm{Kr}(E=35 \mathrm{AMeV})+{ }^{112} \mathrm{Sn}$. The telescope was mounted at an angle of $16.4^{\circ}$ in the laboratory system, beyond the grazing angle. The $\Delta E-E$ scatter plot of measured ions is presented in fig. 5 with a zoom on the region of the lowest deposited energies. The theoretical energy loss calculation - based on refs. [18, 19 _ - for the most populated isotope of mass $A$ for every

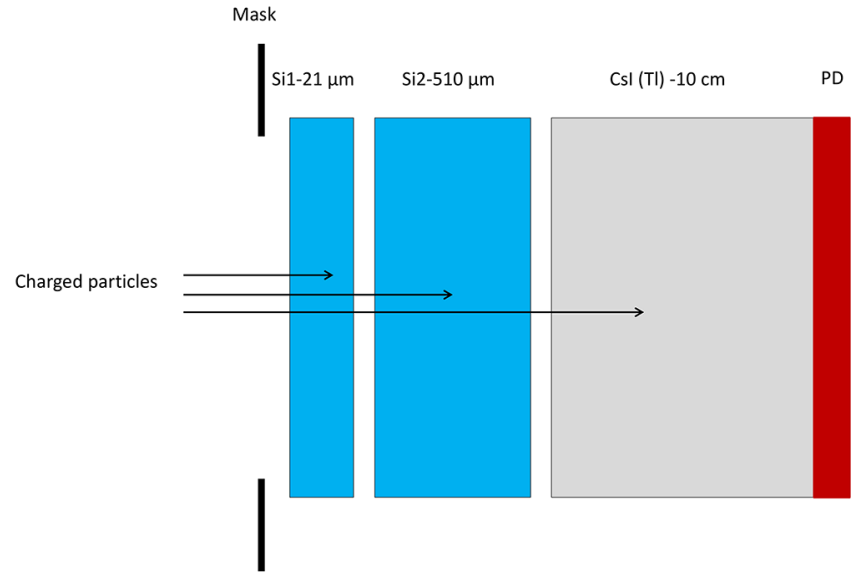

Fig. 4. (Color online) Geometry of the three stage telescope: $\mathrm{Si} 1, \mathrm{Si} 2$ and $\mathrm{CsI}(\mathrm{Tl})$. To remove edge effects, the first detector is collimated with a $20 \mathrm{~mm}$ mask.

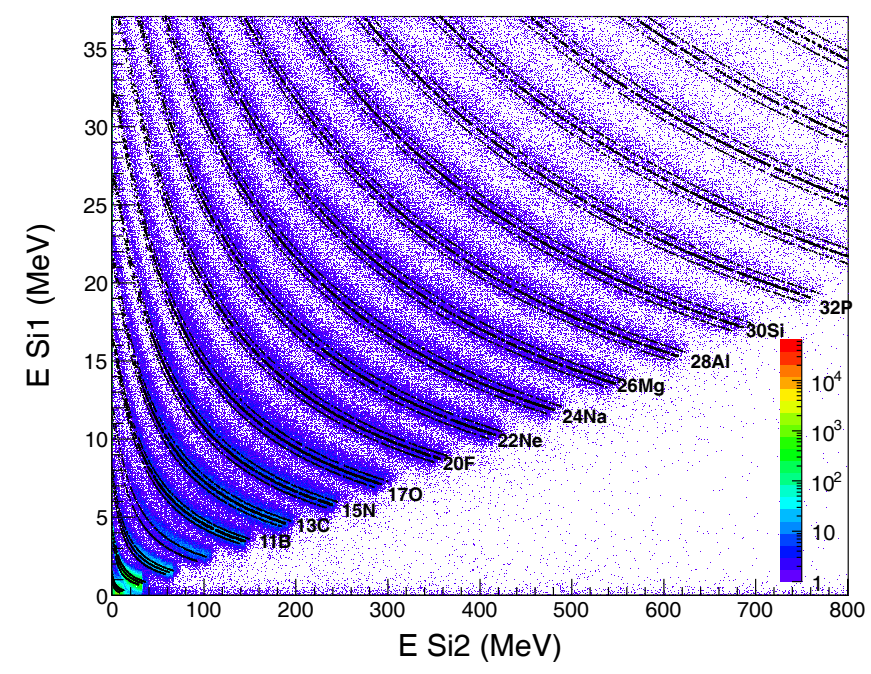

Fig. 5. (Color online) $\Delta E-E$ scatter plot obtained using a $\Delta E(\mathrm{Si} 1)-E(\mathrm{Si} 2)$ telescope, employing our new thin detector as the first stage. A logarithmic scale is used for colors (count levels). Both silicons are rear-mounted according to the FAZIA choice as described in [10]. For a given $Z$, the three black curves indicate the theoretical energy loss calculation for the most populated isotope $A$ and the two adjacent neighbours $A \pm 1$.

given charge $Z$ is shown, surrounded by the ridges calculated for $A \pm 1$. For each element, other isotopes are produced for this system as reported in [20]. During the measurements, the thin detector was biased at around $2-3 \mathrm{~V}$ and the reverse current resulted to be around $1.4 \mu \mathrm{A}$, in agreement with the voltage-current characteristic shown in fig. 3. Since the thin detector has no protection $\mathrm{SiO}_{2}$ layer, a large leakage current is observed. The $\mathrm{Si} 2$ detector was operated at about 290 volt, just beyond the depletion voltage and its reverse current was $20 \mathrm{n} A$. The energy calibration of the $\Delta E(\mathrm{Si} 1)-E(\mathrm{Si} 2)$ telescope was obtained by selection of the punch-through points for several ions identified in charge. Since isotopic resolution is not achieved, for calibration purposes we rely on the most populated 


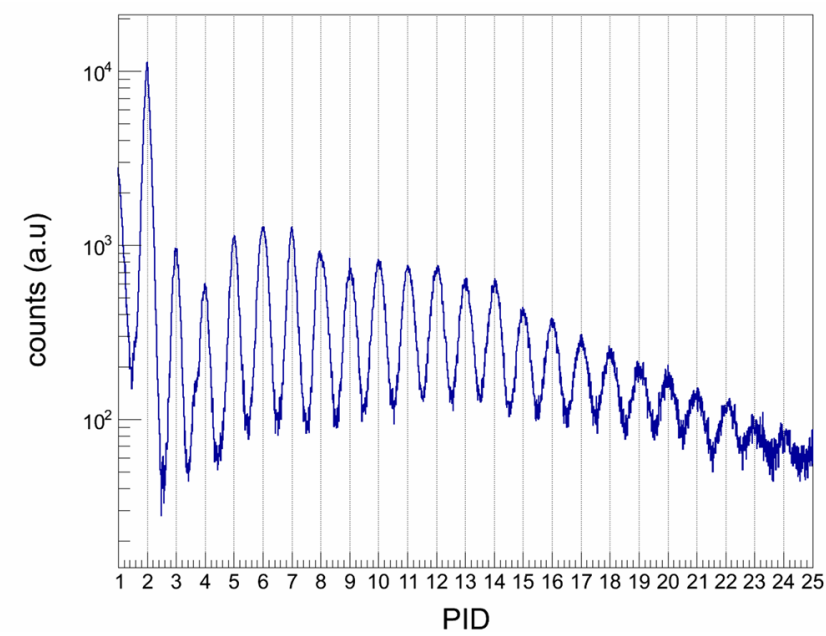

Fig. 6. Particle identification distribution (PID) of measured ions obtained after lineralization of the $\Delta E(\mathrm{Si} 1)-E(\mathrm{Si} 2)$ matrix shown in fig. 5 . The peaks correspond to the various ion charges.

isotopes as found in the same experiment with a standard FAZIA telescope. As a matter of fact, we associated the punch-through points to the calculated energies $[18,19]$ for the ions: ${ }^{4} \mathrm{He},{ }^{7} \mathrm{Li},{ }^{9} \mathrm{Be},{ }^{11} \mathrm{~B},{ }^{13} \mathrm{C},{ }^{15} \mathrm{~N},{ }^{17} \mathrm{O},{ }^{20} \mathrm{~F},{ }^{22} \mathrm{Ne}$, ${ }^{24} \mathrm{Na},{ }^{26} \mathrm{Mg},{ }^{28} \mathrm{Al},{ }^{30} \mathrm{Si},{ }^{32} \mathrm{P},{ }^{34} \mathrm{~S}$, which stop in the second $E(\mathrm{Si} 2)(510 \mu \mathrm{m})$ detector. Calibrations for thin (Si1) and thick (Si2) detectors of the telescope are linear versus energy for all registered heavy ions. The ridges appearing in the $\Delta E-E$ plot have been linearized with purposely dedicated software, already developed in the past by the INDRA Collaboration [21], in order to get a particle identification (PID) parameter. The threshold, corresponding to the (Si1) thickness, varies between 1.1 and $2 \mathrm{AMeV}$, when $Z$ varies between 2 and 25 . The PID distribution is presented in fig. 6 for all measured ions and shows $Z$ identification from helium to manganese. Hydrogen is not well identified as the relative locus in the $\Delta E-E$ plot is very close to the noise limit.

Since the FAZIA Collaboration has deeply investigated the PSA as a powerful tool to identify particles stopped in a given silicon layer, we tried to apply this technique at this new thin detector. The results are shown in fig. 7, which reports the identification plots $E(\operatorname{Si1})$ versus $Q$ risetime (upper) and $E(\mathrm{Si1})$ versus $I_{\max }$ (lower) for ions stopped in the thin Si1. A small cluster of events induced by an electronic pulse generator is present in both panels. These plots are those commonly studied and used by the FAZIA Collaboration as explained in [9-11]. It appears that no separation can be obtained for the various ions. This result is not unexpected considering the previous findings and arguments also proposed in our previous papers. Indeed, it has been shown since pioneering works on PSA [22] that ion identification is lost when ions have short ranges in silicon (i.e., low energies). This experimental observation was reproduced by our simulations $[1,23$, $24]$, as well. Although very thin large-area detectors appear to be inappropriate for PSA applications, their development is of extreme interest for the heavy-ion research
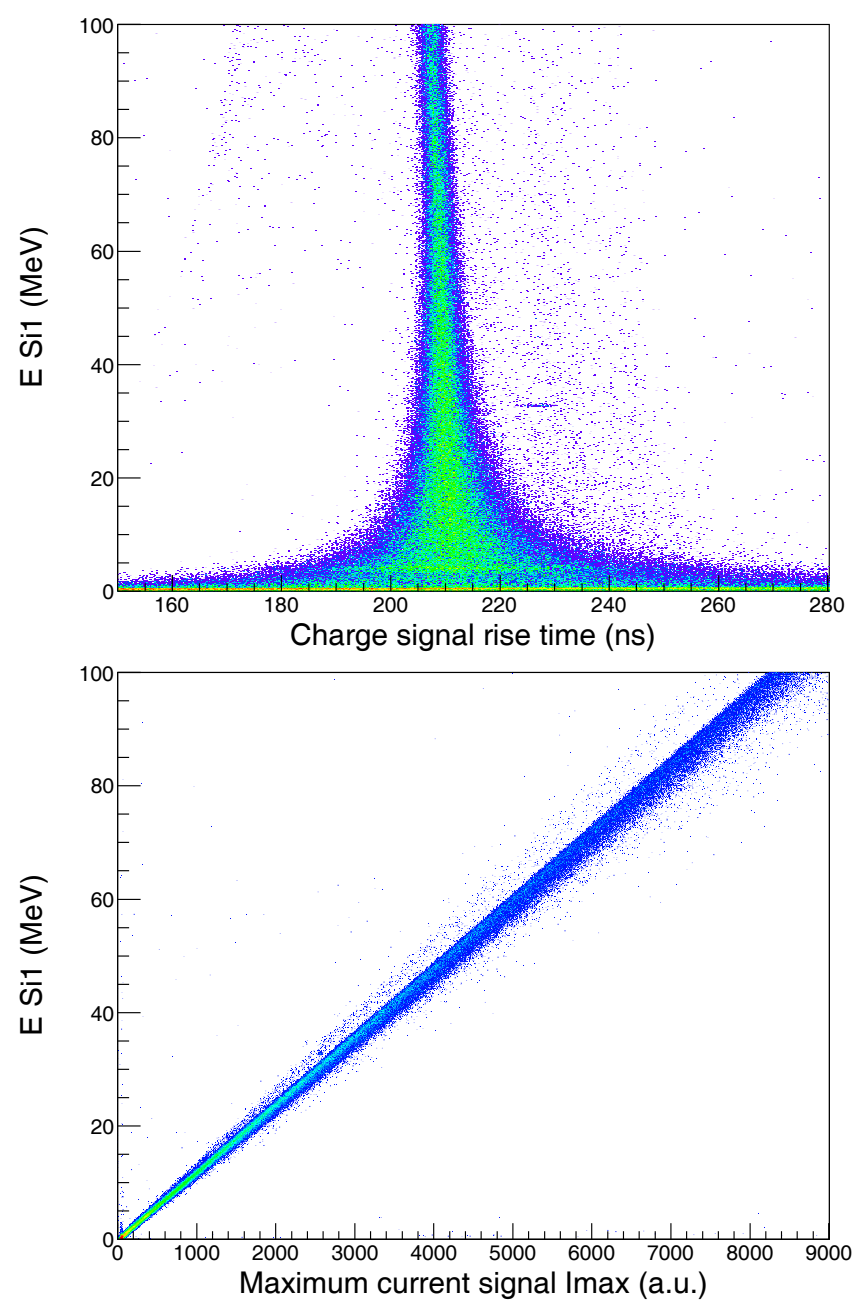

Fig. 7. (Color online) Energy versus $Q$-risetime (upper panel) and energy versus $I_{\max }$ (lower panel) for the thin detector. See text for explanation.

at low energies, as those foreseen at the next radioactive beam ISOL laboratories like SPES and SPIRAL-2. In fact, although FAZIA demonstrated [1] that PSA is capable to sizeably reduce the identification thresholds for ions stopped in rather thick silicon detectors, however, PSA still represent a limit for the charge separation of many reaction products at bombarding energies well below the Fermi regime. In this respect the development of very thin and flat silicon detectors is important for the complementary recovery of the $\Delta E-E$ technique.

The essence of the present new technique is an application of the low-temperature baking process for postimplantation thermal treatment instead of the hightemperature annealing process used elsewhere. The new technique, thanks to the moderate temperatures, can be applied also after the evaporation of metal contacts on both sides of the detector. An additional achievement of the technique is the use of a common mask for both $\mathrm{B}^{+}$ ion implantation and $\mathrm{Al}$ evaporation on the junction side of the detector. As the $\mathrm{B}^{+}$implantation takes place after the anodic dissolution, this technology would allow manufacturing strip detectors too. 


\section{Summary and conclusions}

A new method to construct thin silicon detectors has been implemented and a large-area pioneer silicon pad has been produced and tested under beam. It was mounted as the first stage in a $\Delta E-E$ telescope producing very encouraging results. Indeed, it allowed charge identification in the range $Z=2-25$, for energies higher than 1.1 up to $2 A \mathrm{MeV}$, depending on the ion charge. These very low thresholds are comparable to those introduced by thin ionisation chambers, with the huge advantage of the operation simplicity in case of the silicon detector.

We acknowledge support by the Foundation for Polish Science - MPD program, co-financed by the European Union within the European Regional Development Fund.

Open Access This is an open access article distributed under the terms of the Creative Commons Attribution License (http://creativecommons.org/licenses/by/4.0), which permits unrestricted use, distribution, and reproduction in any medium, provided the original work is properly cited.

\section{References}

1. R. Bougault et al., Eur. Phys. J. A 50, 47 (2014).

2. L. Bardelli et al., Nucl. Instrum. Methods A 605, 353 (2009).

3. S. Barlini et al., Nucl. Instrum. Methods A 707, 89 (2013).

4. L. Bardelli et al., Nucl. Instrum. Methods A 602, 501 (2009).
5. H. Hamrita et al., Nucl. Instrum. Methods A 531, 607 (2004).

6. L. Bardelli et al., Nucl. Instrum. Methods A 521, 480 (2004).

7. G. Pasquali et al., Nucl. Instrum. Methods A 570, 126 (2007).

8. G. Pasquali et al., Nucl. Instrum. Methods A 572, 882 (2007).

9. L. Bardelli et al., Nucl. Instrum. Methods A 654, 272 (2011).

10. S. Carboni et al., Nucl. Instrum. Methods A 664, 251 (2012).

11. N. Le Neindre et al., Nucl. Instrum. Methods A 701, 145 (2013).

12. E. Leagsgaard, Nucl. Instrum. Methods 169, 93 (1979).

13. J. Kemmer, Nucl. Instrum. Methods 169, 449 (1980).

14. A.J. Kordyasz et al., Nucl. Instrum. Methods A 570, 336 (2007).

15. L. Lavergne-Gosselin et al., Nucl. Instrum. Methods A 276, 210 (1989).

16. L. Stab, Nucl. Instrum. Methods A 288, 24 (1990).

17. A.J. Kordyasz et al., Nucl. Instrum. Methods A 539, 262 (2005).

18. L.C. Northcliffe R.F. Schilling, At. Nucl. Data Tables A 7, 223 (1970).

19. F. Hubert, R. Bimbot, H. Gauvin, Nucl. Data Tables 46, 1 (1990).

20. G. Pasquali et al., Eur. Phys. J. A 50, 86 (2014).

21. E. De Filippo et al., Rapport Dapnia-SphN-95-60 (1995).

22. G. Paush et al., IEEE Trans. Nucl. Sci. 44-3, 1040 (1997).

23. L. Bardelli et al., in Proceedings of the International Workshop on Multifragmentation IWM2005 (2005) 75.

24. Z. Sosin, Nucl. Instrum. Methods A 693, 170 (2012). 